\title{
Studies on Heterosis in Oat
}

\author{
Charupriya Chauhan*, S.K. Singh and Sanghamitra Rout \\ Department of Genetics and Plant Breeding, Sardar Vallabhbhai Patel University of \\ Agriculture \& Technology, Meerut - 250 110, India \\ *Corresponding author
}

\section{A B S T R A C T}

\begin{tabular}{|l|}
\hline Key w or d s \\
Avena sativa, \\
Heterosis
\end{tabular}

\section{Introduction}

Oat (Avena sativa L. $2 \mathrm{n}=6 \mathrm{x}=42$ ) a constituent of family Poaceae ranks sixth in the world cereal production. It is cultivated for use as food, feed and fodder. The crop has been adopted well by the farmers because of its multicut nature and high yield of nutritious palatable fodder. Oat is a crop of Mediterranean origin, the domestication dates back to ancient times. Oat seeds are reportedly found in 4000 year old remains in Egypt. It is a descended form of member of diploid (14 chromosomes) and a tetraploid wild species. The genus Avena comprises about seventy species, a few are cultivated. Oat belong to genus Avena established by Tournefort, a French explorer and botanist classified it as, Phylum spermatophyta; Sub class
Monocotyledons; Family- Poaceae (Gramineae); Tribe-Avenue and Genus-Avena. There are two divisions of the genus Avena; annual and perennial. Predominantly two species i.e., Avena sativa L. and Avena byzantina $\mathrm{C}$. Koch are under cultivated at global level. The Avena sativa $\mathrm{L}$. is more important, contributing over 80 per cent of the total world acreage. The cultivated oats (Avena sativa L.) a natural allopolyploid, together with wild weedy hexaploid species like Avena sterillis and Avena fatua have evolved through repeated cycles of interspecific hybridization and polyploidization, combining three distinct diploid genomes. All the hexaploid species have genomic constitution of AACCDD. It is well established that the domesticated oats (Avena sativa L.) has evolved from the wild 
hexaploid species Avena sterillis, which is corroborated by the fact that the hybrids between two are fully fertile. The species with chromosome number $2 \mathrm{n}=28$ or 42 , though apparently polyploidy do not normally from multivalent whose presence indicates autopolyploidy or effective homology. Cultivated species of oats are mostly hexaploids, while the wild species, which could contribute valuable genes, are mostly diploid and tetraploid.

\section{Materials and Methods}

The experimental material for the present investigation was comprised of eight promising diverse parents namely Kent, OS6, JHO-99-2, JHO851, OS403, OL125, UPO212 and NDO612 and their all possible $28 \mathrm{~F}_{1}$ s, developed through crossing eight parental lines in diallel mating design (excluding reciprocals). All genotypes were evaluated in a complete randomized block design with three replications at Crop Research Center (Chirodi) of Sardar Vallabhbhai Patel University of Agriculture and Technology, Meerut, during rabi season 2015-16 and 20162017. The observations were recorded on ten characters viz., days to $50 \%$ flowering, number of leaves per plant, leaf area, plant height, number of tillers per plant, stem girth, number of green pods per plant, length of spike, leaf stem ratio and green fodder yield per plant.

The data recorded on all these traits were subjected to various statistical and biometrical analyses viz., to work out analysis of variance, components of variance through numerical approaches, general and specific combining ability variances and effects. The experiment for raising parents and $\mathrm{F}_{1}$ ' $\mathrm{s}$ was conducted in a complete randomized block design with three replications. Seeds of eight parents and $\mathrm{F}_{1}$ s were sown by hand dibbling method and the length of each row was kept $3 \mathrm{~m}$ with inter and intra row distances of $30 \mathrm{~cm}$ and $10 \mathrm{~cm}$, respectively. The data were compiled and subjected to analysis for heterosis expressed as percentage increase or decrease of $\mathrm{F}_{1}$ 's over better parent as well as mid parent was suggested by Fonesca and Patterson (1968).

\section{Results and Discussion}

The result of manifestation of heterosis was found in both positive and negative direction (Table 1) for days to $50 \%$ flowering. The heterosis over better parent ranged from 13.17 (JHO851 x OS403) to 15.85 (UPO212 x NDO612). Out of $28 \mathrm{~F}_{1}$ hybrids, seven crosses observed highly significant and positive heterobeltiosis values viz., Kent x JHO851, Kent x UPO212, JHO-99-2 x UPO212, OS403 $x$ UPO212, OS403 x NDO612, OL125 x UPO212 and UPO212 x NDO612 for late flowering while, one cross combination JHO851 x OS403 exhibited highly significant negative heterosis over better parent for early flowering. Similar results on the importance of positive heterosis for days to $50 \%$ flowering have been reported by Mishra et al., (2014).

Number of leaves per plant magnitude of heterosis varied from -13.56 (JHO-99-2 x OL125) to 32.51 (UPO212 $\mathrm{x}$ NDO612). Eleven crosses i.e. Kent x JHO-99-2, Kent $\mathrm{x}$ JHO851, Kent x OS403, Kent x UPO212, OS6 x JHO-99-2, JHO-99-2 x JHO851, JHO851 x OL125, JHO851 x UPO212, OS403 x OL125, OL125 x NDO612 and UPO212 x NDO612 noted positive and significant heterosis over better parent for more number of leaves per plant and hybrid JHO-99-2 x OL125 recorded negative significant heterosis over better parent for this character. Estimates of positive heterosis for this character were earlier reported by Dumlupinar et al., (2015). Heterobeltiosis for leaf area was found in the ranged -17.48 (JHO851 $\mathrm{x}$ OS403) to 24.04 (JHO-99-2 x OL125) of heterobeltiosis was recorded among all the $F_{1}$ 's for stem girth. 
Table.1 Estimates of heterosis (\%) over better parent (Heterobeltiosis) and mid parent (Relative heterosis) of yield and its components in oats (Avena sativa L.)

\begin{tabular}{|c|c|c|c|c|c|c|c|c|c|c|}
\hline \multirow[t]{2}{*}{ Hybrids } & \multicolumn{2}{|c|}{ Days to $50 \%$ flowering } & \multicolumn{2}{|c|}{ No. of leaves per plant } & \multicolumn{2}{|c|}{ Leaf area $\left(\mathrm{cm}^{2}\right)$} & \multicolumn{2}{|c|}{ Plant height $(\mathrm{cm})$} & \multicolumn{2}{|c|}{ No. of tillers per plant } \\
\hline & BP & MP & BP & MP & BP & MP & $\mathbf{B P}$ & MP & BP & MP \\
\hline Kent x OS6 & 3.40 & 2.45 & -6.12 & -6.51 & -1.05 & -5.11 & 4.88 & 2.57 & $-13.46^{* *}$ & $-12.48 * *$ \\
\hline Kent x JHO-99-2 & 5.56 & 3.64 & $17.43 * *$ & $16.76^{* *}$ & 5.24 & 4.79 & 0.66 & 0.67 & $15.38 * *$ & $14.76^{* * *}$ \\
\hline Kent x JHO 851 & $14.06 * *$ & $13.30 * *$ & $18.97 * *$ & $18.33^{* *}$ & $14.22 * *$ & $10.43 * *$ & 0.63 & 0.91 & $24.18 * *$ & $23.81 * *$ \\
\hline Kent x OS 403 & 5.86 & 2.54 & $23.54 * *$ & $22.39 * *$ & 4.50 & 3.49 & 3.54 & 1.66 & -2.33 & -1.40 \\
\hline Kent x OL 125 & 4.01 & 1.66 & -5.80 & -4.46 & -1.60 & -2.03 & 0.94 & 0.13 & $-14.36 * *$ & $-10.02 * *$ \\
\hline Kent x UPO 212 & $7.07 * *$ & $6.41 * *$ & $24.38 * *$ & $23.90 * *$ & $12.44 * *$ & $12.27 * *$ & $15.00 * *$ & $14.29 * *$ & $17.38 * *$ & $16.88^{* *}$ \\
\hline Kent x NDO 612 & 4.01 & 4.01 & 6.78 & 5.37 & 2.60 & 1.17 & 3.56 & 2.83 & $-11.64 * *$ & $-10.15 * *$ \\
\hline OS 6 x JHO-99-2 & 1.82 & 0.90 & $26.31 * *$ & $20.38 * *$ & 2.05 & 1.96 & 5.60 & 5.85 & 3.87 & 4.90 \\
\hline OS-6 x JHO 851 & -4.13 & -1.71 & -2.37 & -2.11 & -0.08 & -0.94 & 2.00 & 1.61 & -3.06 & -3.47 \\
\hline OS $6 \times$ OS 403 & 3.64 & 1.33 & 1.08 & 1.02 & -3.17 & -1.37 & 2.05 & 1.57 & 1.15 & 1.97 \\
\hline OS $6 \times$ OL 125 & -1.82 & -0.45 & 1.03 & 1.01 & 1.25 & 1.94 & 3.56 & 1.01 & $14.01 * *$ & $13.62 * *$ \\
\hline OS 6 x UPO 212 & 4.74 & 3.07 & -3.97 & -3.16 & -0.82 & -0.81 & $-1.50 *$ & -1.91 & $-18.96 * *$ & $-17.18 * *$ \\
\hline OS 6 x NDO 612 & 1.85 & 0.92 & -1.58 & $28.00 * *$ & 0.91 & 0.17 & -1.76 & 3.72 & 1.50 & 1.29 \\
\hline JHO-99-2 x JHO 851 & 1.62 & 1.15 & $19.05 * *$ & $18.63 * *$ & $16.36^{* *}$ & $16.12 * *$ & $14.72 * *$ & $13.36 * *$ & $24.96 * *$ & $23.08 * *$ \\
\hline JHO-99-2 x OS 403 & -1.79 & -0.44 & $-10.86^{* *}$ & $-10.11 * *$ & -4.42 & -3.14 & $15.08 * *$ & $13.31 * *$ & 3.14 & 3.90 \\
\hline JHO-99-2 x OL 125 & 2.08 & 1.63 & $-13.56^{* *}$ & $-13.49 * *$ & $24.04 * *$ & $23.44 * *$ & 3.74 & 2.51 & -2.40 & -4.26 \\
\hline JHO-99-2 x UPO 212 & $12.45^{* *}$ & $11.53 * *$ & -2.84 & 1.97 & 6.83 & 2.31 & $-18.77 * *$ & $-17.09 * *$ & 4.82 & 3.81 \\
\hline JHO-99-2 x NDO 612 & 4.01 & 2.12 & -3.32 & -2.66 & -0.66 & -0.10 & 1.74 & 1.66 & $18.78^{* *}$ & $17.72 * *$ \\
\hline JHO 851 x OS 403 & $-13.17 * *$ & $-12.52 * *$ & -1.54 & 1.06 & $-17.48 * *$ & $-17.11 * *$ & 5.07 & 4.85 & -2.95 & -1.05 \\
\hline JHO 851 x OL 125 & 3.49 & -0.31 & $18.26^{* * *}$ & $17.13 * *$ & -4.45 & -2.98 & 4.32 & 3.04 & 5.62 & 4.50 \\
\hline JHO 851 x UPO 212 & 1.38 & 1.29 & $22.60 * *$ & $22.00 * *$ & -1.77 & -1.87 & 5.12 & 4.06 & 4.18 & 2.27 \\
\hline JHO 851 x NDO 612 & 1.59 & 0.16 & 3.12 & 3.01 & -1.53 & -1.42 & 1.95 & 0.53 & $-14.02 * *$ & $-13.52 * *$ \\
\hline OS $403 \times$ OL 125 & 2.06 & 1.17 & $20.58 * *$ & $19.71 * *$ & $-14.30 * *$ & $-13.49 * *$ & 4.14 & 3.05 & 1.54 & 2.11 \\
\hline OS 403 x UPO 212 & $14.47 * *$ & $13.92 * *$ & 7.55 & 6.85 & -2.40 & -2.29 & $-17.95^{* *}$ & $-16.44 * *$ & -1.40 & -2.84 \\
\hline OS403 x NDO 612 & $7.41 * *$ & $7.04 * *$ & -2.80 & -2.34 & 2.25 & 1.74 & 2.70 & 1.59 & -1.99 & -1.49 \\
\hline OL 125 x UPO 212 & $12.12 * *$ & $11.72 * *$ & 3.61 & 3.01 & 1.28 & 1.75 & 2.77 & 1.69 & 5.46 & 4.63 \\
\hline OL 125 x NDO 612 & 4.94 & 2.56 & $18.36 * *$ & $17.28 * *$ & 3.08 & 2.88 & 0.92 & 0.86 & 6.57 & 5.73 \\
\hline
\end{tabular}




\begin{tabular}{|c|c|c|c|c|c|c|c|c|c|c|}
\hline UPO 212 x NDO 612 & $15.05 * *$ & $14.48 * *$ & $32.51 * *$ & $31.84 * *$ & $15.35 * *$ & $14.36 * *$ & $16.66 * *$ & $14.35 * *$ & $19.03 * *$ & $18.48 * *$ \\
\hline SE & 2.15 & 1.61 & 1.00 & 0.74 & 0.68 & 0.51 & 2.91 & 2.06 & 0.29 & 0.25 \\
\hline Hybrids & \multicolumn{2}{|c|}{ Stem girth (mm) } & \multicolumn{2}{|c|}{$\begin{array}{c}\text { No. of green pods per } \\
\text { spike }\end{array}$} & \multicolumn{2}{|c|}{ Length of spike (cm) } & \multicolumn{2}{|c|}{ Leaf stem ratio (w/w) } & \multicolumn{2}{|c|}{$\begin{array}{c}\text { Green fodder yield per } \\
\text { plant (g) }\end{array}$} \\
\hline & BP & MP & BP & MP & BP & MP & BP & MP & BP & MP \\
\hline Kent x OS6 & -0.24 & -0.93 & 0.70 & 0.71 & -4.14 & -1.70 & -3.35 & -3.38 & 5.36 & 4.53 \\
\hline Kent x JHO-99-2 & 5.00 & 4.49 & 0.29 & 0.19 & -1.24 & -0.32 & 0.68 & 0.50 & $19.03 * *$ & $18.24 * *$ \\
\hline Kent x JHO 851 & $7.67 * *$ & $6.76 * *$ & $17.48 * *$ & $17.14 * *$ & $7.00 * *$ & $6.00 * *$ & $20.53 * *$ & $20.50 * *$ & $29.98 *$ & $29.70 * *$ \\
\hline Kent x OS 403 & -2.86 & -2.78 & $-12.59 * *$ & $-11.50 * *$ & -1.84 & 1.72 & $11.05 * *$ & $10.62 * *$ & $13.76^{* *}$ & $13.39 * *$ \\
\hline Kent x OL 125 & 4.48 & 3.83 & $-23.13 * *$ & $-20.03 * *$ & -4.35 & -3.14 & -2.15 & -1.92 & $11.21 * *$ & $11.00 * *$ \\
\hline Kent x UPO 212 & $20.19 * *$ & $19.16 * *$ & $36.51 * *$ & $35.71 * *$ & $21.14 * *$ & $20.03 * *$ & 0.56 & 0.96 & $39.99 * *$ & $38.94 * *$ \\
\hline Kent x NDO 612 & 4.22 & 4.56 & -3.56 & -1.14 & 0.60 & 0.20 & $-12.73 * *$ & $-11.08 * *$ & $17.08 * *$ & $16.98 * *$ \\
\hline OS 6 x JHO-99-2 & $-19.28 * *$ & $-18.45^{* *}$ & -6.39 & -5.25 & 5.56 & 4.58 & 3.18 & 2.78 & 1.85 & 1.75 \\
\hline OS-6 x JHO 851 & -2.24 & -1.28 & 5.16 & 2.76 & 0.52 & 0.42 & $14.31 * *$ & $13.91 * *$ & -0.91 & -0.69 \\
\hline OS 6 x OS 403 & 2.03 & 1.33 & -0.30 & -0.26 & 1.29 & 1.96 & $23.34 * *$ & $22.44 * *$ & 5.80 & 5.72 \\
\hline OS 6 x OL 125 & -5.61 & -4.31 & $-27.93 * *$ & $-26.76 * *$ & 1.85 & 3.89 & -2.87 & -1.41 & -1.83 & 1.74 \\
\hline OS 6 x UPO 212 & -4.65 & -5.01 & -2.48 & -1.81 & 5.01 & 5.70 & -1.12 & -1.92 & $14.46^{* *}$ & $13.50 * *$ \\
\hline OS 6 x NDO 612 & $-18.64 * *$ & $-18.39 * *$ & -0.51 & -0.37 & 4.70 & 3.65 & $16.85 * *$ & $15.21 * *$ & 3.06 & 2.76 \\
\hline JHO-99-2 x JHO 851 & $14.87 * *$ & $14.59 * *$ & $26.39 * *$ & $25.80 * *$ & $24.17 * *$ & $23.79 * *$ & $38.91 * *$ & $34.93 * *$ & $27.71 * *$ & $27.53 * *$ \\
\hline JHO-99-2 x OS 403 & -5.35 & -4.64 & $-17.02 * *$ & $-17.00 * *$ & $-16.45 * *$ & $-15.46^{* *}$ & $13.45 * *$ & $12.55^{* *}$ & $18.58 * *$ & $17.55 * *$ \\
\hline JHO-99-2 x OL 125 & -1.21 & -1.14 & $-14.01 * *$ & $-13.96 * *$ & -4.62 & -3.85 & 0.52 & 0.17 & 5.19 & 4.34 \\
\hline JHO-99-2 x UPO 212 & 3.44 & 3.30 & -4.28 & -3.46 & -3.42 & -1.85 & 1.22 & 1.07 & 3.24 & 2.77 \\
\hline JHO-99-2 x NDO 612 & $17.12 * *$ & $16.08 * *$ & -3.87 & -2.06 & 1.60 & 1.06 & 1.28 & 1.86 & $-17.82 * *$ & $-16.14 * *$ \\
\hline JHO 851 x OS 403 & 2.71 & 1.91 & $13.69 * *$ & $12.73 * *$ & 1.08 & 1.34 & 5.04 & 4.15 & $15.25^{* *}$ & $14.30 * *$ \\
\hline JHO 851 x OL 125 & -1.04 & -0.67 & 3.42 & 1.13 & -1.04 & 1.46 & 2.96 & 1.45 & 2.84 & 1.38 \\
\hline JHO 851 x UPO 212 & -4.55 & -3.02 & $-20.11 * *$ & $-19.18 * *$ & -4.45 & -1.39 & -4.02 & -3.00 & 7.05 & 6.24 \\
\hline JHO 851 x NDO 612 & -3.07 & 2.95 & -3.91 & -2.16 & 1.50 & 0.90 & 4.61 & 3.49 & $15.50 * *$ & $11.37 * *$ \\
\hline OS $403 \times$ OL 125 & 0.04 & 0.21 & -4.52 & -3.19 & -1.22 & -0.93 & $23.86 * *$ & $22.64 * *$ & $12.75^{* * *}$ & $12.34 * *$ \\
\hline OS 403 x UPO 212 & -4.27 & -2.66 & $-17.48 * *$ & $-16.41 * *$ & $-11.21 * *$ & $-10.57 * *$ & $18.24 * *$ & $16.39 * *$ & $11.99 * *$ & $11.89 * *$ \\
\hline OS403 x NDO 612 & 3.41 & 2.74 & 2.77 & 1.12 & 5.47 & 4.60 & $30.98 * *$ & $29.40 * *$ & 1.98 & 1.63 \\
\hline OL 125 x UPO 212 & 1.84 & 1.67 & 4.82 & 2.57 & -3.75 & -2.46 & 3.95 & 2.80 & 1.08 & 1.40 \\
\hline OL 125 x NDO 612 & 4.28 & 3.30 & 5.16 & 3.32 & $22.36^{* * *}$ & $20.15 * *$ & -4.27 & -3.41 & $14.60 * *$ & $16.69 * *$ \\
\hline UPO 212 x NDO 612 & $19.83 * *$ & $18.36 * *$ & $24.74 * *$ & $23.31 * *$ & $15.40 * *$ & $16.50 * *$ & $14.55^{* *}$ & $13.47 * *$ & $38.40 * *$ & $37.85 * *$ \\
\hline SE & 0.15 & 0.12 & 1.28 & 0.92 & 0.68 & 0.51 & 0.01 & 0.01 & 3.02 & 2.89 \\
\hline
\end{tabular}


The cross combinations viz., Kent x JHO851, Kent x UPO212, JHO-99-2 x JHO851, JHO 99-2 x OL 125 and UPO212 x NDO612 showed positive significant heterosis over better parent for leaf area and two crosses JHO851 x OS403 and OS403 x OL125 revealed negative significant heterosis over better parent for this character. The present study is in agreement with Kapoor and Bajaj (2013). A range for plant height per plant was -18.77 (JHO-99-2 x UPO212) to 16.66 (UPO212 x NDO612). The best crosses i.e. Kent x UPO212, JHO-99-2 x JHO851, JHO99-2 x OS 403 and UPO212 x NDO612 exhibited positive and significant heterosis over better parent, suggested that good performance for this character and two hybrids JHO-99-2 x UPO212 and OS403 x UPO212 revealed negative and significant heterosis over better parent, indicated poor performance for plant height. These results are somewhat in accordance with the findings of Dumlupinar et al., (2015). Heterobeltiosis for number of tillers per plant over the better parent ranged from -18.96 (OS6 x UPO212) to 24.96 (JHO-99-2 x JHO851). Out of twenty eight $\mathrm{F}_{1}$ 's hybrid, only seven crosses namely, Kent x JHO-99-2, Kent x JHO851, Kent x UPO212, OS6 $x$ OL 125, JHO-99-2 x JHO851, JHO-99-2 x NDO612 and UPO212 $x$ NDO612 showed positive and significant heterosis in order to merit over better parent and five crosses revealed negative significant heterosis over better parent for this trait. Such types of findings were reported by Vishwakarma et al., (2010). Magnitude of heterosis for stem girth varied from -19.28 (OS6 x JHO-99-2) to 20.19 (Kent x UPO212). Hybrids viz., Kent $\mathrm{x}$ JHO851, Kent $\mathrm{x}$ UPO212, JHO-99-2 x JHO851, JHO-99-2 x NDO612 and UPO212 x NDO612 exhibited positive significant heterosis over better parent for stem girth and only two $\mathrm{F}_{1}$ 's OS6 x JHO-99-2 and OS6 x NDO612 showed negative significant heterosis over better parent, emerged as poor performance for this character. Positive heterosis for stem girth studied has also been reported earlier Mishra et al., (2014) and Dumlupinar et al., (2015). Heterobeltiosis for number of green pods per spike ranged from -27.93 (OS6 x OL125) to 36.51 (Kent $x$ UPO212). Five $F_{1}$ 's hybrid noted highly significant and positive heterosis over better parent values in Kent x JHO851, Kent x UPO212, JHO-99-2 x JHO851, JHO851 x OS403 and UPO212 x NDO612. Seven crosses recorded significant negative heterosis over better parent for number of green pods per plant. Similar results were found by Verma and Singh (2010). The degree of heterosis over better parent for length of spike varied from -16.45 (JHO-99-2 $\mathrm{x}$ OS403) to 24.17 (JHO-99-2 x JHO851). Highly significant and positive heterosis values over better parent were recorded in five $\mathrm{F}_{1}$ hybrids viz., Kent $\mathrm{x}$ JHO851, Kent $\mathrm{x}$ UPO212, JHO-99-2 x JHO851, OL125 x UPO212 and UPO212 x NDO612 for length of spike and two crosses JHO-99-2 x OS403 and OS403 x UPO212 noted negative and significant heterosis over better parent for this character. Heterosis of similar magnitude has been reported by Vishwakarma et al., (2010). Estimates of heterosis for leaf stem ratio ranged from -12.73 (Kent $x$ NDO612) to 38.91 (JHO-99-2 x JHO851). A total of $11 \mathrm{~F}_{1}$ hybrids revealed positive and highly significant heterosis over better parent, these were Kent x JHO851, Kent x OS403, OS6 x JHO851, OS6 x OS403, OS6 x NDO612, JHO-99-2 x JHO851, JHO-99-2 x OS403, OS403 x OL125, OS403 x UPO212, OS403 x NDO612 and UPO212 x NDO612. Only one cross Kent $x$ NDO612 had significant and negative heterosis over better parent for leaf stem ratio. These results are in conformity with the findings of Dumlupinar et al., (2015). Expression of heterosis over better parent for green fodder yield per plant varied from - 17.82 (JHO-99-2 x NDO612) to 39.99 (Kent x UPO212). Out of 28 F' 's, 15 crosses showed highly significant and positive 
heterobeltiosis values, viz., Kent x JHO-99-2, Kent $\mathrm{x}$ JHO851, Kent $\mathrm{x}$ OS403, Kent $\mathrm{x}$ OL125, Kent x UPO212, Kent x NDO612, OS6 x UPO212, JHO-99-2 x JHO851, JHO99-2 x OS403, JHO851 x OS403, JHO851 x NDO612, OS403 x OL125, OS403 $\mathrm{x}$ UPO212, OL $125 \times$ NDO612 and UPO $212 x$ NDO612 for green fodder yield per plant, whereas, only one cross combination JHO-99$2 \times$ NDO612 exhibited negative significant heterosis over better parent for this character. The expression of heterosis of similar magnitude for this attribute has also been reported by Kapoor and Bajaj (2013), Mishra et al., (2014) and Dumlupinar et al., (2015). As above cross combinations may be exploited for heterosis breeding programme. Since these hybrids involve high $\mathrm{x}$ high or high $\mathrm{x}$ average or average $\mathrm{x}$ average or average $x$ low or low $x$ low gca value of parent and significant sca effect for involved of non-additive gene action and response of dominance and dominance $\mathrm{x}$ dominance type of gene effect. A high heterosis result for yield might be obtained by exploiting these individual cross for developing hybrids through heterosis breeding programme.

\section{References}

Dumlupinar, Z., Karakuzulu, H., Demirtas, M.B., Ugurer, M., Gezginc, H.,
Dokuyucu, T. and Akkaya, A. (2015). A Heterosis study for some agronomic traits in oat. Journal of Agricultural Sciences 21: 414-419.

Fonseca, S. and Patterson, F. (1968).Hybrid vigour in a seven parent diallel crosses in common winter wheat. (Linum usitatissimum L.).Crop Sci., 8: 85-88.

Kapoor, R. and Bajaj, R. K. (2013). Combining ability and heterosis studies in Avena sativa L. for green fodder yield and component traits. Vegetos, 26(1): 272-277.

Mishra, P., Arora, R.N., Joshi, U.N., and Chhabra, A.K. (2014). Heterosis and combining ability for quality traits in intervarietal and interspecific hybrids in oat. Forage Research., 40(2): 86-90.

Verma, J.S. and Singh, G.(2010).Combining Ability effects and heterosis for grain yield and grain protein content in oat (Avena sativa L.). National symposium on "Forage Resource and livestock for livelihood, Environment and Nutritional Security" September 10-11, 2011. Range Management society of India, IGFRI, Jhansi.

Vishwakarma, D.N., Ram C.N. Chauhan, S.S., Vishwakama, S.R. and Maurya, M.L. (2010). Heterosis for green forage and grain yield characters in oat. Agric. Boil. Res., 26: 77-81.

\section{How to cite this article:}

Charupriya Chauhan, S.K. Singh and Sanghamitra Rout. 2018. Studies on Heterosis in Oat. Int.J.Curr.Microbiol.App.Sci. 7(09): 272-277. doi: https://doi.org/10.20546/ijcmas.2018.709.034 